\title{
MR Neurography of the Lumbosacral Plexus for Lower Extremity Radiculopathy: Frequency of Findings, Characteristics of Abnormal Intraneural Signal, and Correlation with Electromyography
}

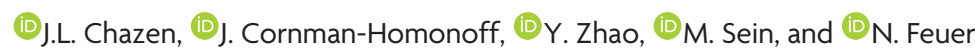

\begin{abstract}
BACKGROUND AND PURPOSE: MR neurography enables high resolution imaging of peripheral nerves. Our aim was to evaluate the utility of MR neurography in lumbosacral radiculopathy and correlate abnormal intraneural signal with history, physical examination, and abnormal electrodiagnostic study findings.

MATERIALS AND METHODS: Retrospective review of lumbosacral MR neurography examinations performed from December 2014 through January 2017 on a 3T scanner was undertaken. MR neurography examinations were independently reviewed in a blinded fashion by 2 radiologists, and the intraneural signal was graded on a $0-2$ scale relative to adjacent vasculature. Abnormal nerve signal was correlated with subjective and objective findings from clinical notes in the electronic medical record and compared with results of electrodiagnostic studies (nerve conduction study/electromyography).

RESULTS: Three hundred three lumbosacral MR neurography examinations were performed during the study period, 64 of which met the inclusion criteria, including symptoms of radiculopathy on electromyography performed within 3 months of MR neurography. Twentynine (45\%) MR neurography examinations had abnormal intraneural signal. There was no statistically significant correlation between subjective clinical findings and intraneural signal abnormality on MR neurography. There was a statistically significant correlation between abnormal intraneural T2 signal and findings of active radiculopathy on electromyography $(P<.001)$.

CONCLUSIONS: Lumbosacral MR neurography appears to demonstrate abnormal intraneural signal in a substantial portion of patients with clinical symptoms of lower extremity radiculopathy and correlates with findings of active radiculopathy on electromyography. This finding further bolsters the growing body of evidence on the utility of MR neurography and suggests that abnormal intraneural signal may provide a useful adjunct to electrodiagnostic testing. Further research is required to evaluate the prognostic value of MR neurography, which may help guide therapeutic decision-making.
\end{abstract}

ABBREVIATIONS: EMG = electromyography; MRN = MR neurography; MUAP = motor unit action potential; NCS = nerve conduction study; SPAIR = spectral adiabatic inversion recovery

umbosacral radiculopathy has a prevalence of approximately 3\%-5\% in the general population. ${ }^{1}$ It affects men and women equally, though the onset in men is, on average, a decade earlier than in women. ${ }^{1}$ Clinical evaluation relies predominantly on history and physical examination. Electrodiagnostic testing including needle electromyography (EMG) has been described as an "extension" of the clinical examination but remains an imperfect

Received April 17, 2018; accepted after revision July 27.

From the Departments of Radiology (J.L.C.), Resident Physician (J.C.-H.), Rehabilitation Medicine (M.S), and Neurology (N.F.), Center for Comprehensive Spine Care, Weill Cornell Medicine, NewYork-Presbyterian Hospital, New York, New York; and Department of Healthcare Policy \& Research (Y.Z.), Weill Cornell Medicine, New York, New York.

Please address correspondence to J. Levi Chazen, MD, Weill Cornell Medicine, NewYork-Presbyterian Hospital, Department of Radiology, 525 East 68th St, Starr 631, New York, NY 10065; e-mail: jlc2008@med.cornell.edu

http://dx.doi.org/10.3174/ajnr.A5797 test with a sensitivity ranging from $49 \%$ to $86 \%$ for lumbosacral radiculopathy. ${ }^{2,3}$ Despite its limitations, EMG can be a useful adjunct examination, given the high rate of false-positive findings on spinal MR imaging in asymptomatic individuals. ${ }^{4,5}$

MR neurography (MRN), first described in 1992, is a novel technique increasingly used in clinical practice to evaluate extraspinal neuropathy. ${ }^{6}$ The MRN technique relies on high-field-strength magnets, anisotropic diffusion along the nerve path, and fat-suppressed T2-based imaging with long TEs to optimize intraneural signal. Endoneural fluid and T2 signal are increased with nerve irritation and compression. ${ }^{7}$ A variety of neuropathies can be evaluated with MRN, including neoplastic, traumatic, and inflammatory/autoimmune disease. ${ }^{8} \mathrm{MRN}$ of extraspinal nerve entrapment has generated particular interest. In an investigation by Lewis et al, ${ }^{9}$ abnormal sciatic nerve signal and findings of entrapment were seen in 12 of 14 patients on MRN, with sciatica symptoms and unrevealing lumbar spine MR 


\begin{tabular}{|c|c|c|c|c|c|c|c|c|}
\hline Sequence & Matrix & TR (ms) & TE (ms) & NEX & $\begin{array}{l}\text { FOV } \\
(\mathrm{cm})\end{array}$ & $\begin{array}{l}\text { In-Plane Resolution } \\
(\mathrm{mm})\end{array}$ & $\begin{array}{l}\text { Slice Thickness } \\
(\mathrm{mm})\end{array}$ & $\begin{array}{l}\text { Acquisition Time } \\
\text { (min: sec) }\end{array}$ \\
\hline Axial T1 TSE & $320 \times 240$ & 663 & 12 & 2 & 31 & $1.0 \times 1.3$ & 3.5 & $6: 18$ \\
\hline Axial T2 FS SPAIR & $320 \times 192$ & 6220 & 83 & 2 & 31 & $1.0 \times 1.6$ & 3.5 & $7: 52$ \\
\hline Coronal T1 & $384 \times 216$ & 3160 & 9.9 & 1 & 36 & $1.0 \times 1.7$ & 3 & $3: 49$ \\
\hline Coronal PD SPAIR & $320 \times 224$ & 4380 & 35 & 2 & 36 & $1.1 \times 1.6$ & 3 & $4: 10$ \\
\hline 3D Coronal SPACE STIR & $256 \times 256$ & 1500 & 119 & 1.4 & 38 & $1.5 \times 1.5$ & 1.5 & $8: 23$ \\
\hline Sagittal T2 Dixon (lumbar spine) & $448 \times 300$ & 4160 & 83 & 2 & 28 & $0.6 \times 0.9$ & 3 & $3: 59$ \\
\hline
\end{tabular}

Note:-PD indicates proton density; SPACE, sampling perfection with application-optimized contrasts by using different flip angle evolution (Siemens); FS, fat suppressed. ${ }^{a}$ All lumbosacral plexus MR imaging was performed on a 3T Magnetom Skyra (Siemens) scanner without contrast.

December 2014 - January 2017: 303 lumbosacral MRN reviewed

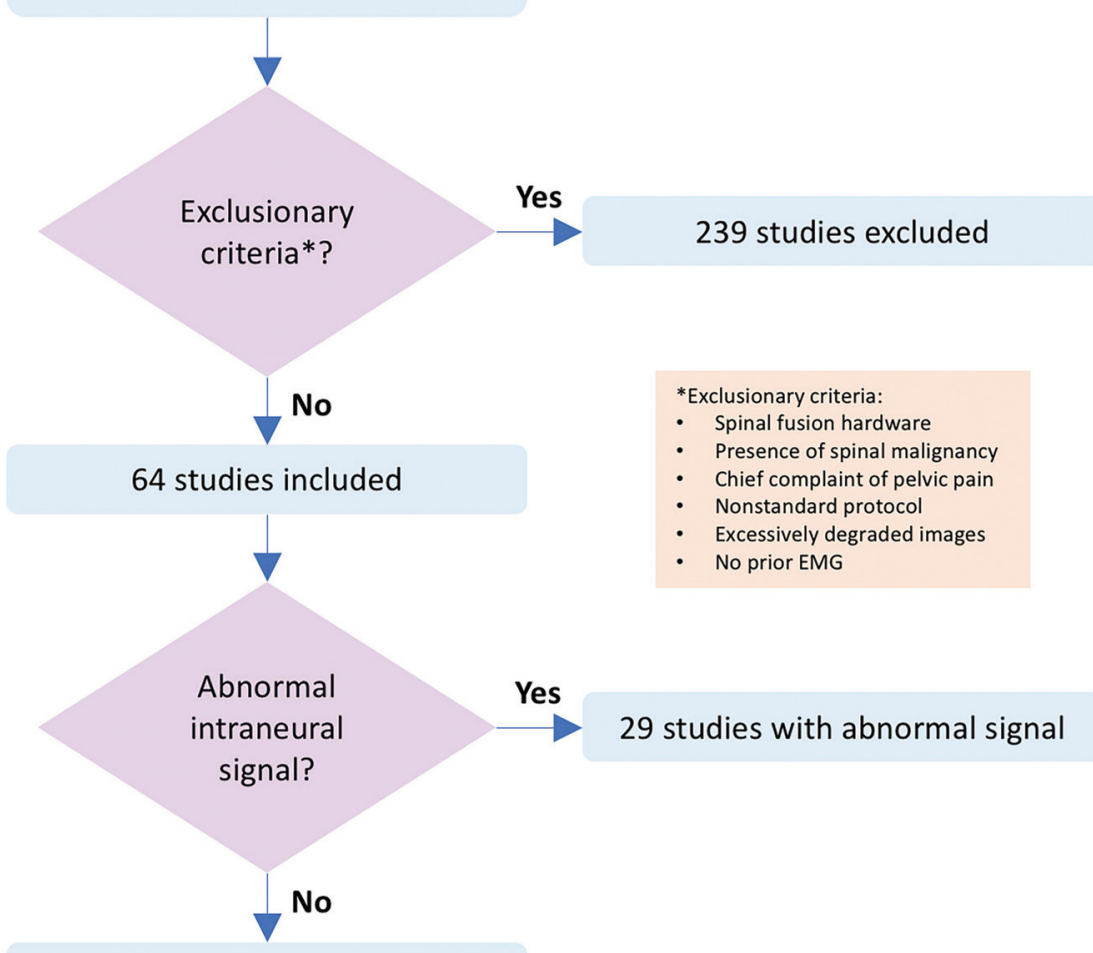

35 studies with normal signal lopathy and correlates MRN abnormalities with those described on EMG. MRN can precisely identify abnormal signal in extraspinal nerves as they exit the neuroforamen and traverse anatomic corridors to join the lumbosacral plexus. This extraspinal signal abnormality may allow more accurate and specific evaluation of abnormal nerves responsible for clinical symptoms than traditional methods. Furthermore, extraspinal extraneural causes for symptomatology (eg, hip osteoarthritis masquerading as high lumbar "radicular" symptoms) can be identified at the time of MRN.

\section{MATERIALS AND METHODS Study Participants}

Weill Cornell Medicine institutional review board approval was obtained for this retrospective study with a waiver of informed consent. Patients included in the analysis had clinical signs of active lower extremity radiculopathy and both a MR neurogram and EMG performed within a 3-month timeframe at our institution. Patients were excluded if they had spinal fusion hardware, which could limit the MR neurogram evaluation from susceptibility artifact.

FIG 1. Flowchart illustrating study selection.

\section{MR Imaging Protocol and Evaluation}

MR imaging was performed at 3T on a Magnetom Skyra platform (Siemens, Erlangen, Germany). The MRN imaging protocol is described in Table 1 and consisted of limited sagittal lumbar spine imaging along with axial and coronal $\mathrm{T} 1$ and fat-suppressed $\mathrm{T} 2$ sequences through the lumbosacral plexus. Imaging studies were evaluated by a radiologist with a Certificate of Added Qualification in neuroradiology (J.L.C.), and scores were recorded for statistical analysis. Following a 10-case training session, MRN examinations were independently evaluated by a radiology senior resident (J.C.-H.) to determine interrater reliability. The radiologists were blinded to clinical history, including the laterality of pain. Abnormal nerves were graded on a categoric scale from $0-2$ with 0 representing normal signal; 1 , increased intraneural signal but less than adjacent vasculature; and 2, marked increased intraneural signal similar to that in adjacent vascular structures. If multiple abnormal nerves were iden- 
Table 2: Demographics/clinical characteristics and MRN/EMG findings

\begin{tabular}{lcc}
\hline \multicolumn{1}{c}{ Variable } & Value & $\begin{array}{c}\text { Range/ } \\
\text { Percentage }\end{array}$ \\
\hline Subjects & 64 & \\
Age (yr) & 57 & $20-84$ \\
Sex (male) & 33 & $51.6 \%$ \\
Symptom duration (mo) & 26 & $1-312$ \\
Diabetes & 12 & $18.8 \%$ \\
Prior surgery & 9 & $14.1 \%$ \\
Weakness & 29 & $45.3 \%$ \\
Numbness & 31 & $48.4 \%$ \\
Leg pain & 48 & $75.0 \%$ \\
Back pain & 28 & $43.8 \%$ \\
Tingling & 23 & $35.9 \%$ \\
MRN intraneural T2 signal abnormality & 28 & $43.8 \%$ \\
$\quad$ (grade I or II) & & \\
MRN evidence of muscular denervation & 8 & $12.5 \%$ \\
EMG findings of active radiculopathy & 20 & $31.3 \%$ \\
\hline
\end{tabular}

tified, the most pronounced signal abnormality was recorded and the proximal nerve was considered for analysis (for example, if the ipsilateral L4 and femoral nerves were abnormal, it was graded as an L4 radiculopathy).

Imaging findings of muscular denervation were rated as positive if T2 hyperintense edema was present in the substance of the paraspinous, psoas, iliacus, gluteal, piriformis, adductor, or proximal thigh musculature.

\section{Nerve Conduction Studies/EMG Protocol and Evaluation}

Nerve conduction studies (NCSs) and needle EMG were performed by board-certified neuromuscular neurologists at our institution. Reports were individually reviewed by an experienced neuromuscular neurologist (N.F.). Nerve conduction studies included standard tested sensory (superficial peroneal and sural) and motor (peroneal and tibial) nerves in the legs. These were analyzed by measuring the waveform latency, amplitude, duration, and conduction velocity. Late responses were also evaluated, including the F-Responses and H-reflex, to aid in the evaluation of the proximal nerve segments.

Needle electromyography was performed using a concentric needle in leg muscles representing L2-S1 myotomes. Muscles were evaluated for the presence of active denervation, motor unit action potential (MUAP) morphology, and MUAP recruitment and activation patterns. When present, active denervation was determined by fibrillation potentials and positive sharp waves. Chronic re-innervation changes in a muscle were defined by the decreased activation and recruitment of neurogenic (long duration and high amplitude) MUAPs.

\section{Electronic Medical Record Review}

The electronic medical records of the included subjects were reviewed for subjective findings of lower extremity weakness, numbness, leg pain, back pain, tingling, burning, pain quality, and severity (Visual Analog Scale 1-10). Patient history was recorded, including the inciting event, history of surgery, diabetes mellitus, and symptom duration. Physical examination findings were collected, including the presence of atrophy, weakness, decreased sensation, decreased reflexes, and positive findings on straight leg raises.
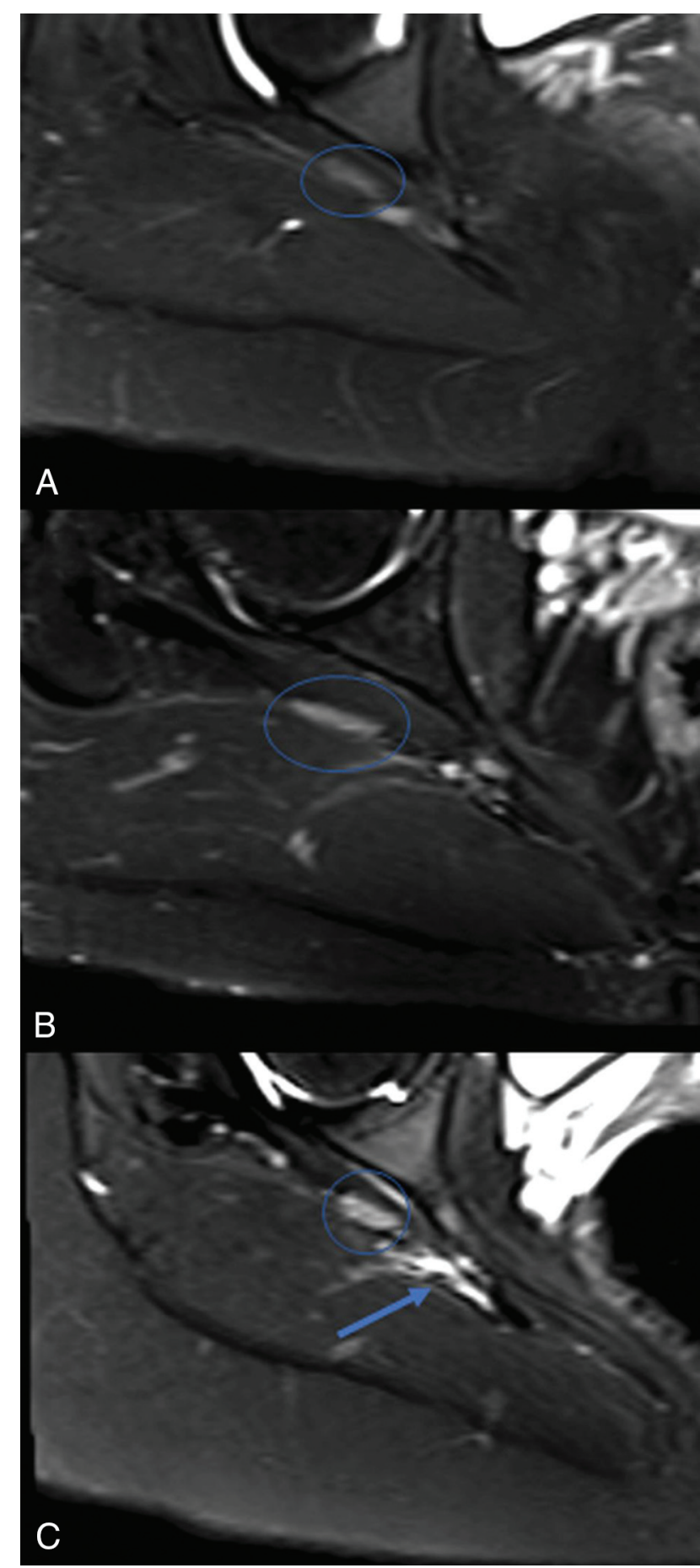

FIG 2. Axial fat-suppressed T2-weighted spectral adiabatic inversion recovery (SPAIR) images just distal to the greater sciatic foramen. $A$, Grade 0 nerve signal in a normal right sciatic nerve (circle). B, Grade I nerve signal abnormality shows a mildly hyperintense right sciatic nerve but less intense than the adjacent vasculature. C, Grade II nerve signal abnormality, similar to that of adjacent veins.

\section{Statistical Analysis}

Demographic variables and MR imaging parameters were summarized by mean/SD (continuous variables) and frequency/percentage (categoric variables). The comparison between ordinal variables (ie, signal, in 2 groups) was achieved with a Wilcoxon rank sum test. The association between categorical variables was 


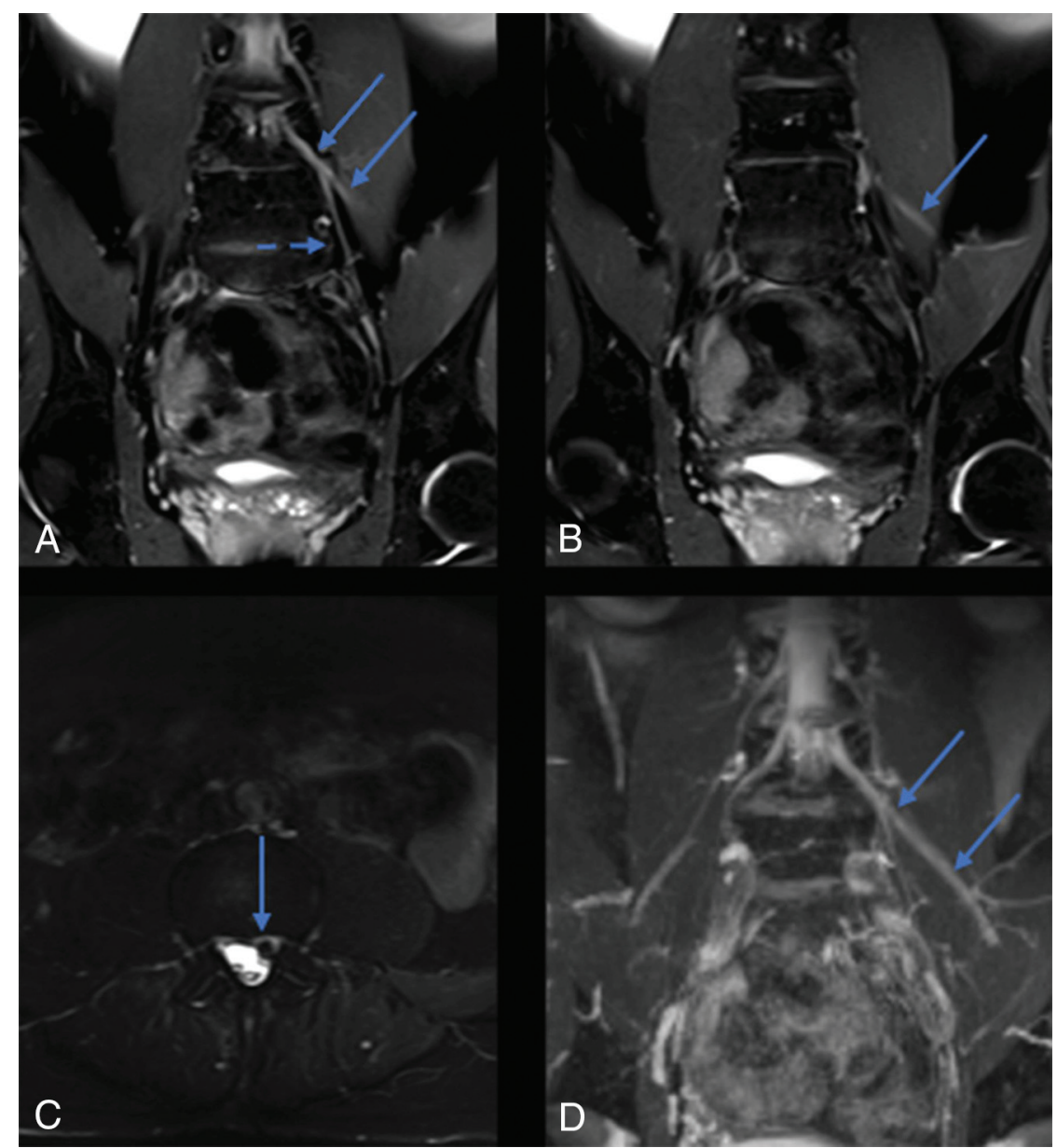

FIG 3. Coronal fat-suppressed T2 SPAIR ( $A$ and $B)$, axial fat-suppressed T2 SPAIR $(C)$, and maximum-intensity reconstruction of 3D coronal fat-suppressed T2 STIR (D) reveal marked abnormal asymmetric signal involving the left $\mathrm{L} 4$ nerve root extending to the left femoral nerve (arrows, $A$, $B$, and $D$ ). Signal abnormality also extends along the left obturator nerve (dashed arrow, $A$ ), also supplied by the L4 nerve root. An axial slice through the L3-L4 level (C) shows a disc extrusion (arrow, C) compressing the left L4 nerve root, accounting for the nerve inflammation.

\section{Table 3: Statistical association}

\begin{tabular}{lcc}
\hline \multicolumn{1}{c}{ Correlations } & $\begin{array}{c}\boldsymbol{\varphi} \text { Coefficient/ } \\
\text { Cramér V }\end{array}$ & $\boldsymbol{P}$ Value \\
\hline Denervation/subjective weakness & 0.13 & .30 \\
Denervation/objective weakness & 0.21 & .10 \\
Denervation/objective atrophy & 0.33 & .02 \\
MRN intraneural signal/EMG active & 0.79 & $<.001$ \\
$\quad$ radiculopathy & & \\
MRN laterality/EMG laterality & 1.00 & $<.001$ \\
\hline
\end{tabular}

measured by the $\varphi$ coefficient under a Pearson $\chi^{2}$ test or a Fisher exact test. A $\kappa$ statistic was calculated for interrater reliability between the 2 interpreters of MRN examinations. All the statistical analyses were performed by R statistical and computing software, Version 3.4.3 (http://www.r-project.org/). For the statistical tests, relevant $P$ values are reported with a significant level of .05 .

\section{RESULTS}

Three hundred three MRN studies were performed during the study period from December 2014 through January 2017. Of these, 64 patients were included in the final analysis when both MR neurograms and EMG were acquired within a 3-month timeframe and exclusion criteria were not met (Fig 1). The mean sub- ject age was 57 years (range, 20-84 years), $52 \%$ were male, and $75 \%$ reported leg pain. Demographics and clinical characteristics are described in Table 2. Half of the patients had decreased lower extremity sensation on physical examination, and 36\% showed some signs of objective weakness. On MRN, 28 of 64 patients $(44 \%)$ of the cohort had at least 1 nerve with abnormal intraneural signal on the fat-suppressed T2weighted images (Fig 2). Of the 28 patients with abnormal intraneural T2 signal, 18 (64\%) had a grade 2 signal abnormality, similar to that in adjacent vasculature (Fig 3). Eight patients (13\%) had MRN findings of active muscular denervation. On NCS and EMG evaluations, 20 of 64 patients (31\%) had findings of an active radiculopathy with denervation changes (Table 2). There was substantial agreement between readers with a $\kappa$ statistic of 0.71 (confidence boundary, $0.41,1$ ) on blinded MRN interpretation. Ten subjects with suspicious findings on MRN had normal electrodiagnostic test findings without evidence of active radiculopathy.

Abnormal intraneural signal was not significantly associated with subjective clinical abnormalities. The association between patient-reported symptom duration, weakness, numbness, leg pain, back pain, or tingling and abnormal intraneural signal on MRN was not significant. Similarly, no significant association was identified between physician-reported findings of weakness, decreased sensation, decreased deep tendon reflexes, the presence of positive straight leg raises, and abnormal intraneural signal. However, there was a statistically significant correlation between objective findings of muscle atrophy and denervation changes on $\operatorname{MRN}(P=.02)$.

There was a statistically significant association between abnormal intraneural T2 signal and findings of active radiculopathy on EMG with a mean intraneural signal score of 0.3 in patients without findings of active radiculopathy versus 1.7 in patients with EMG findings of active radiculopathy $(P<.001)$. Furthermore, when abnormal intraneural signal was treated as a binary variable (normal or abnormal), there was a statistically significant association between signal abnormality on MRN and active radiculopathy on EMG $(P<.001)$ (Table 3$)$.

\section{DISCUSSION}

This study revealed a statistically significant correlation between abnormal intraneural signal on MRN and findings of active denervation on NCS/EMG performed within 3 months. To our knowledge, this correlation has not been demonstrated previ- 


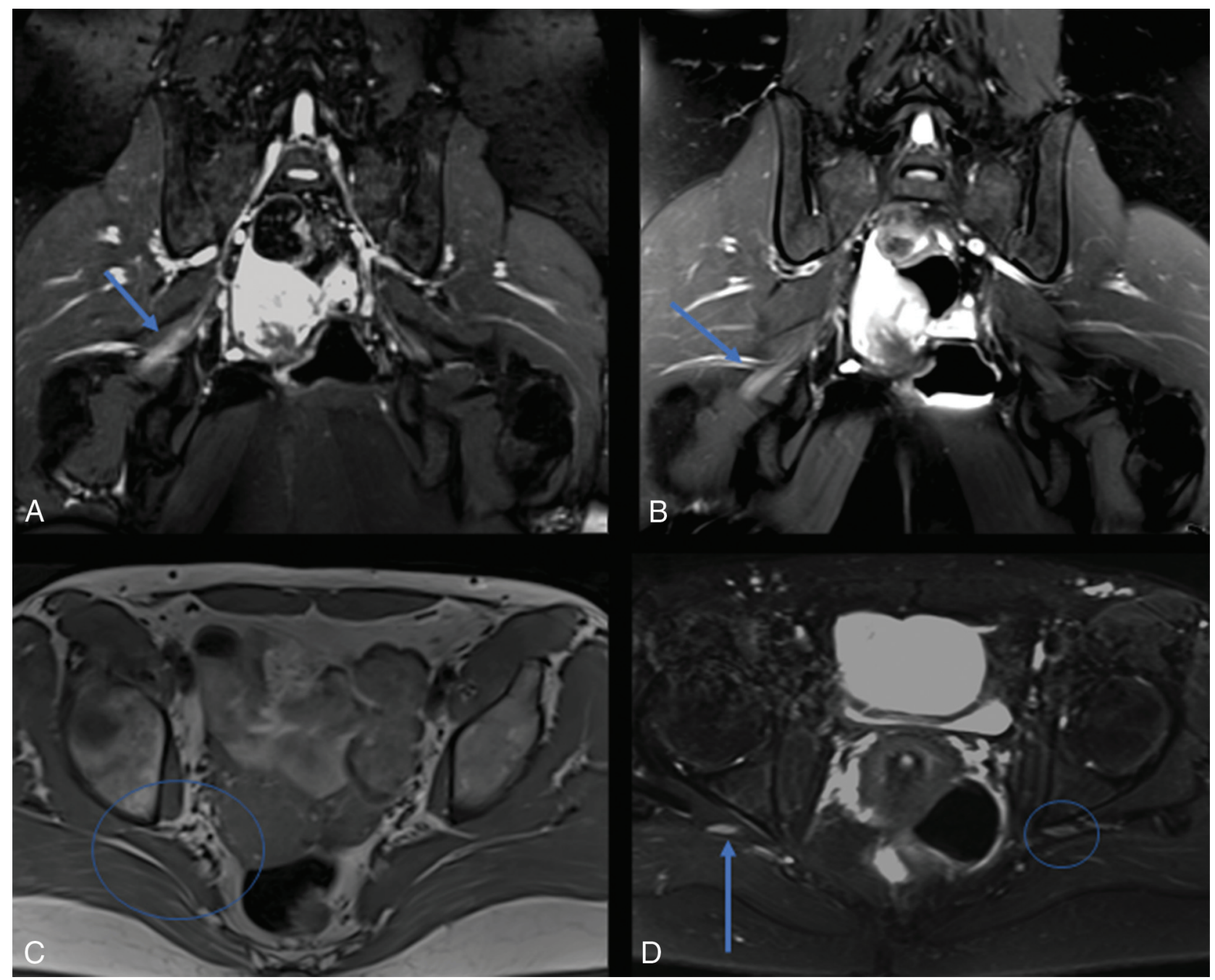

FIG 4. $3 D$ coronal fat-suppressed $T 2 \operatorname{STIR}(A)$, coronal fat-suppressed $T 2$ SPAIR (B), axial $T 1(C)$, and axial fat-suppressed T2 SPAIR (D) images show a typical example of right sciatic neuropathy. There is abnormal signal in the right sciatic nerve (arrows) through the greater sciatic foramen (circle, $C$ ), a characteristic location of piriformis-related sciatic nerve compression. There is corresponding asymmetric signal of the right sciatic nerve compared with the left (circle, $D$ ).

ously for lumbosacral radiculopathy. Furthermore, this imagingelectrodiagnostic correlation was one of the only statistically significant findings in our cohort despite the consideration of multiple subjective and physical examination findings. Lumbar radiculopathy is notoriously difficult to diagnose with history or physical examination findings alone. ${ }^{13,14}$ MRN appears to provide useful diagnostic information, and the statistical correlation with EMG further reinforces its accuracy.

A study by Crim and Ingalls ${ }^{15}$ revealed a moderate sensitivity $(41.2 \%-70.6 \%)$ and very high specificity $(97.7 \%-100 \%)$ in the interpretation of brachial plexus MRN when EMG was used as a criterion standard. This study was not limited to radiculopathy and included MRN studies performed at both 1.5T and 3T. A study by Chhabra et al ${ }^{16}$ revealed a measurable impact of 3T MRN on diagnostic thinking and therapeutic choices. MRN is a recommended study for presurgical planning in patients with peripheral neuropathy. The observed interrater reliability $(\kappa=0.71)$ demonstrates substantial agreement between readers, in line with studies of lumbar spine imaging. ${ }^{17}$

This study evaluated the intraneural T2 signal as a marker for nerve abnormality on MRN. The authors do not find nerve caliber to be a useful metric in this setting because the abnormal nerves in this cohort had a size similar to that of normal nerves (Figs 2 and 4). Furthermore, it is difficult to reproducibly measure the caliber of nerve fascicles, given their oblique orientation, while excluding the perineural fat and soft tissues. Findings of acute or subacute muscular denervation were recorded when $\mathrm{T} 2$ hyperintense signal was seen within the lumbosacral musculature, as described by others. ${ }^{8}$ Imaging findings of muscular denervation were seen in a minority of patients $(12.5 \%)$ but were helpful findings to confirm a neuropathy when observed. For example, in 1 subject with an L4 radiculopathy extending to the femoral nerve, ipsilateral denervation changes were observed in the iliacus, a muscle innervated by the femoral nerve (Fig 5). The relatively low rate of observed denervation changes may be related to the timing of imaging to the active period of radiculopathy and the exclusion of lower thigh and lower extremity muscles from the imaging FOV.

Diffusion-weighted imaging or diffusion tensor imaging was not part of the imaging protocol in this study. The authors have not found that DTI provides useful information when evaluating 


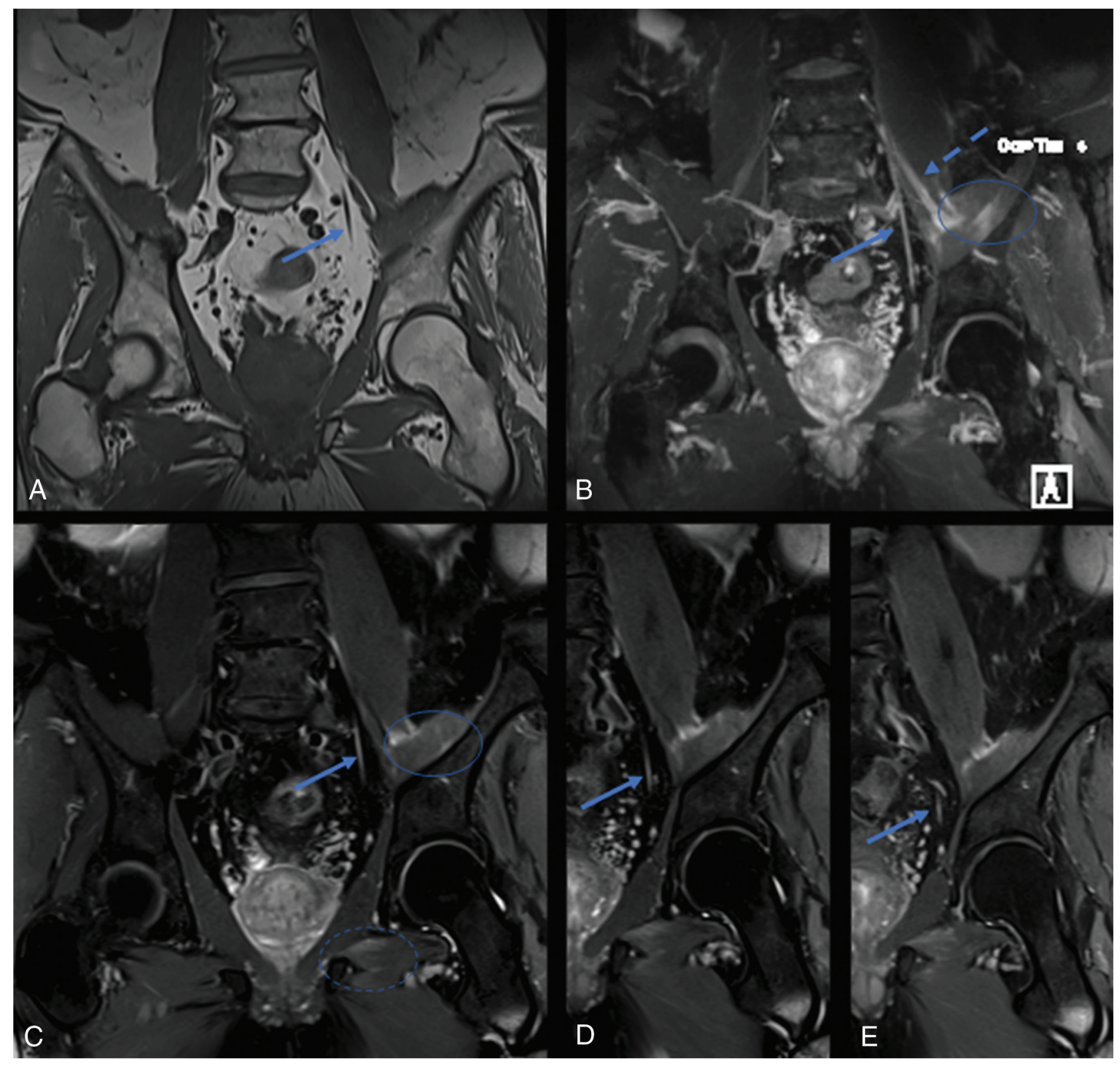

FIG 5. Coronal $T I(A)$, maximum-intensity reconstruction of 3D coronal fat-suppressed $T 2$ STIR $(B)$, and sequential coronal fat-suppressed $T 2$ SPAIR ( $C-E$ ) images show abnormal signal of the left L4, left femoral (dashed arrow, $B$ ) and left obturator (arrows) nerves. There is also abnormal intramuscular T2 hyperintense signal in the left iliacus muscle (circle, B). This patient had a long-standing history of type 2 diabetes, and signal abnormality was attributed to diabetic amyotrophy.

the lumbosacral plexus despite initially acquiring a coronal reversed fast imaging with steady-state precession diffusion sequence as part of the imaging protocol. DWI/DTI may have utility in imaging of peripheral nerves where surface coils can be placed close to the nerves of interest for an improved signal-to-noise ratio. 18

Electrodiagnostic studies are not a perfect criterion standard, and there are variabilities inherent in the performance and interpretation of NCS and EMG. In patients with active radiculopathy, electrodiagnostic studies classically demonstrate a combination of normal NCS and abnormal needle EMG. ${ }^{19}$ This pattern is thought to result from the common lateral recess or subarticular location of a disc herniation proximal to the dorsal root ganglion, thus sparing the sensory nerve fibers. Needle EMG reveals fibril- lation potentials and positive sharp waves when a neuropathic lesion is present. These denervation potentials represent the spontaneous depolarization of an individual muscle fiber from ongoing or active axonal drop-out. The time course of radiculopathy is not always clear on NCS/EMG, but certain findings can narrow the window, including the MUAP morphology. Active denervation may take some time to be apparent on NCS/EMG; it is only evident following axonal injury once Wallerian degeneration begins. Evidence of active denervation with normal MUAP morphology suggests a subacute time course in which the muscles have not yet undergone reinnervation. Regardless, by the time a patient with true radiculopathy comes to clinical attention, there are typically electrodiagnostic findings.

Electrodiagnostic testing has several limitations and may dem- 
onstrate confounding results, for example, when a superimposed peripheral neuropathy or primary myopathy is present. Furthermore, the NCS/EMG is highly operator-dependent, and falsenegative studies with equivocal findings are common despite true radiculopathy for a variety of reasons, including a fascicular phenomenon and variations in electromyographer technique and experience. Despite these limitations, EMG remains a useful examination to evaluate active radiculopathy as class II evidence and level B recommendation by the American Association of Neuromuscular and Electrodiagnostic Medicine in lumbosacral radiculopathy in an analysis of 119 articles. ${ }^{20}$

This study is limited by its retrospective nature and variations in the timing of clinical notes, though all notes reviewed were from neuromuscular neurologists who performed the electrodiagnostic testing. There are also variabilities inherent in MRN interpretation, though the interreader variability was substantial between interpreters. Despite these limitations, it is the largest study performed, to the authors' knowledge, that correlates MRN and electrodiagnostic findings in patients with lumbosacral radiculopathy.

\section{CONCLUSIONS}

In this cohort of patients with lower extremity radiculopathy who had both MRN and electrodiagnostic studies performed within 3 months, $44 \%$ had abnormal intraneural signal on MRN and $31 \%$ had electrodiagnostic findings of active radiculopathy with a statistically significant correlation between positive findings on these modalities. The role of MRN in the work-up and clinical management of patients with lumbosacral radiculopathy is still being explored. The presence of abnormal extraspinal intraneural signal on MRN may identify patients with a high likelihood of response to targeted therapy such as transforaminal epidural steroid injection. It is anticipated that this information will help guide clinical management of patients presenting with active radicular symptoms. These data may prove to be particularly useful in an era of overexuberant epidural injection treatment and personalized medicine with a focus on cost-benefit treatment algorithms.

Disclosures: Michael Sein-UNRELATED: Consultancy: CID Management Solutions.

\section{REFERENCES}

1. Tarulli AW, Raynor EM. Lumbosacral radiculopathy. Neurol Clin 2007;25:387-405 CrossRef Medline

2. Dillingham TR. Evaluating the patient with suspected radiculopathy. PM R 2013;5:S41-49 CrossRef Medline

3. Tong HC, Haig AJ, Yamakawa KS, et al. Specificity of needle electromyography for lumbar radiculopathy and plexopathy in 55- to 79year-old asymptomatic subjects. Am J Phys Med Rehabil 2006;85: 908-12; quiz 913-15, 934 CrossRef Medline
4. Jensen MC, Brant-Zawadzki MN, Obuchowski N, et al. Magnetic resonance imaging of the lumbar spine in people without back pain. N Engl J Med 1994;331:69-73 CrossRef Medline

5. Boden SD, McCowin PR, Davis DO, et al. Abnormal magnetic-resonance scans of the cervical spine in asymptomatic subjects: a prospective investigation. J Bone Joint Surg Am 1990;72:1178-84 CrossRef Medline

6. Howe FA, Filler AG, Bell BA, et al. Magnetic resonance neurography. Magn Reson Med 1992;28:328-38 CrossRef Medline

7. Chhabra A, Flammang A, Padua A Jr, et al. Magnetic resonance neurography: technical considerations. Neuroimaging Clin N Am 2014;24:67-78 CrossRef Medline

8. Soldatos T, Andreisek G, Thawait GK, et al. High-resolution 3-T MR neurography of the lumbosacral plexus. Radiographics 2013;33: 967-87 CrossRef Medline

9. Lewis AM, Layzer R, Engstrom JW, et al. Magnetic resonance neurography in extraspinal sciatica. Arch Neurol 2006;63:1469-72 CrossRef Medline

10. Filler AG, Haynes J, Jordan SE, et al. Sciatica of nondisc origin and piriformis syndrome: diagnosis by magnetic resonance neurography and interventional magnetic resonance imaging with outcome study of resulting treatment. J Neurosurg Spine 2005;2:99-115 CrossRef Medline

11. Yoshida T, Sueyoshi T, Suwazono S, et al. Three-Tesla magnetic resonance neurography of the brachial plexus in cervical radiculopathy. Muscle Nerve 2015;52:392-96 CrossRef Medline

12. Laporte C, Albert JD, Duvauferrier R, et al. MRI investigation of radiating pain in the lower limbs: value of an additional sequence dedicated to the lumbosacral plexus and pelvic girdle. AJR Am J Roentgenol 2014;203:1280-85 CrossRef Medline

13. Iversen T, Solberg TK, Romner B, et al. Accuracy of physical examination for chronic lumbar radiculopathy. BMC Musculoskelet Disord 2013;14:206 CrossRef Medline

14. Suri P, Rainville J, Katz JN, et al. The accuracy of the physical examination for the diagnosis of midlumbar and low lumbar nerve root impingement. Spine 2011;36:63-73 CrossRef Medline

15. Crim J, Ingalls K. Accuracy of MR neurography in the diagnosis of brachial plexopathy. Eur J Radiol 2017;95:24-27 CrossRef Medline

16. Chhabra A, Belzberg AJ, Rosson GD, et al. Impact of high resolution 3 Tesla MR neurography (MRN) on diagnostic thinking and therapeutic patient management. Eur Radiol 2016;26:1235-44 CrossRef Medline

17. Lurie JD, Tosteson AN, Tosteson TD, et al. Reliability of readings of magnetic resonance imaging features of lumbar spinal stenosis. Spine (Phila Pa 1976) 2008;33:1605-10 CrossRef Medline

18. Guggenberger R, Markovic D, Eppenberger P, et al. Assessment of median nerve with MR neurography by using diffusion-tensor imaging: normative and pathologic diffusion values. Radiology 2012;265:194-203 CrossRef Medline

19. Narayanaswami $P$, Geisbush $T$, Jones L, et al. Critically re-evaluating a common technique: accuracy, reliability, and confirmation bias of EMG. Neurology 2016;86:218-23 CrossRef Medline

20. Cho SC, Ferrante MA, Levin KH, et al. Utility of electrodiagnostic testing in evaluating patients with lumbosacral radiculopathy: an evidence-based review. Muscle Nerve 2010;42:276-82 CrossRef Medline 